\title{
Sodium channel mutations in epilepsy and other neurological disorders
}

\author{
Miriam H. Meisler and Jennifer A. Kearney
}

Department of Human Genetics, University of Michigan, Ann Arbor, Michigan, USA.

\begin{abstract}
Since the first mutations of the neuronal sodium channel SCN1A were identified 5 years ago, more than 150 mutations have been described in patients with epilepsy. Many are sporadic mutations and cause loss of function, which demonstrates haploinsufficiency of SCN1A. Mutations resulting in persistent sodium current are also common. Coding variants of $S C N 2 A, S C N 8 A$, and $S C N 9 A$ have also been identified in patients with seizures, ataxia, and sensitivity to pain, respectively. The rapid pace of discoveries suggests that sodium channel mutations are significant factors in the etiology of neurological disease and may contribute to psychiatric disorders as well.
\end{abstract}

\section{Introduction}

Voltage-gated sodium channels are essential for the initiation and propagation of action potentials in neurons. The sodium channel $\alpha$ subunits are large, transmembrane proteins with approximately 2,000 amino acid residues, composed of 4 homologous domains containing well-characterized voltage sensor and pore regions (Figure 1). The transmembrane segments are highly conserved through evolution. The 4 domains associate within the membrane to form a sodium-permeable pore, through which sodium ions flow down a concentration gradient during propagation of an action potential. The transmembrane sodium gradient is subsequently restored by the activity of the ATP-dependent sodium/ potassium pump. The 3 -dimensional structures of related bacterial potassium channels have recently been elucidated $(1,2)$.

Each sodium channel $\alpha$ subunit is associated with 1 or more $\beta$ subunits, $\beta 1$ to $\beta 4$, that are transmembrane proteins with a single extracellular IgG loop and a short intracellular C terminus (Figure 1). Association with $\beta$ subunits influences the level of cell surface expression, voltage dependence, and kinetics of the $\alpha$ subunit, as well as association with other signaling and cytoskeletal molecules $(3,4)$.

Duplication of the $\alpha$ subunit genes during evolution generated 9 mammalian genes encoding active channels that differ in tissue specificity and biophysical properties (Table 1) (5, 6). Many disease mutations have been characterized in the skeletal muscle and cardiac channels, but exploration of the role of the 7 neuronal sodium channels in disease is in an early stage.

\section{$\beta 1$ Subunit mutations and GEFS+}

Generalized epilepsy with febrile seizures plus (GEFS+) (OMIM 604233 ) is a mild, dominantly inherited epilepsy characterized by febrile seizures in childhood progressing to generalized epilepsy in adults $(7,8)$. The first connection between sodium channels and epilepsy was the discovery of a $\beta 1$ subunit mutation in a large Australian family with GEFS+ (9). Affected family members are heterozygous for the missense mutation C121W in the extracellular Ig domain of the $\beta 1$ subunit. The mutant channel promotes cell surface expression of the $\alpha$ subunit but exhibits

Nonstandard abbreviations used: GEFS+, generalized epilepsy with febrile seizures plus; SMEI, severe myoclonic epilepsy of infancy.

Conflict of interest: The authors have declared that no conflict of interest exists.

Citation for this article: J. Clin. Invest. 115:2010-2017 (2005).

doi:10.1172/JCI25466. impaired modulation of sodium channel function and cell adhesion (10). A 5-amino acid deletion in the extracellular domain of $\beta 1$ was subsequently found in a family with febrile seizures and early-onset absence epilepsy (11). Impaired inactivation of sodium channel $\alpha$ subunits is the likely mechanism relating $\beta 1$ mutations to neuronal hyperexcitability in epilepsy.

\section{Inherited and de novo mutations of SCN1A in GEFS+ severe myoclonic epilepsy of infancy}

In 1999, linkage analysis in 2 large families localized a second GEFS+ locus to an interval of chromosome 2 q24 that includes a sodium channel gene cluster $(12,13)$. Sequencing of SCN1A demonstrated that affected individuals are heterozygous for missense mutations in highly evolutionarily conserved amino acid residues, T875M in 1 family and $\mathrm{R} 1648 \mathrm{H}$ in the other (14). Since the initial report, 11 additional SCN1A missense mutations have been reported in GEFS+ families (Figure 2A), approximately $10 \%$ of cases tested (14-25).

In 2001, Peter De Jonghe and colleagues discovered mutations of SCN1A in 7 patients with severe myoclonic epilepsy of infancy (SMEI) (26). This disorder is characterized by early onset, usually within the first 6 months of life, followed by progressive worsening of seizures often accompanied by mental deterioration (OMIM 182389). More than 150 mutations have been identified in children with this disorder (Table 2), approximately $50 \%$ of SMEI patients tested. As in GEFS+, the SMEI patients are heterozygous for the mutant alleles. Among 75 cases in which both parents have been tested, in 69 cases, or $90 \%$, the mutations arose de novo in the affected children.

The mutation spectrum in SMEI differs from that in GEFS+. Approximately half of the SMEI mutations are nonsense or frameshift mutations that result in protein truncation (Figure $2 \mathrm{~B}$ ). The truncation mutations are randomly distributed across the SCN1A protein, including the $\mathrm{N}$-terminal domain, transmembrane segments, cytoplasmic loops, and $\mathrm{C}$ terminus. The remaining SMEI mutations are missense mutations that, as in GEFS+, are concentrated within the transmembrane segments of the protein (Figure 2C). Within the large cytoplasmic loops of the $\alpha$ subunits, the only missense mutations are those located very close to the adjacent transmembrane segments.

The large number of de novo SCN1A mutations in children with SMEI demonstrate the importance of considering mutation in the etiology of neurological disease, even in the absence of a positive family history. 


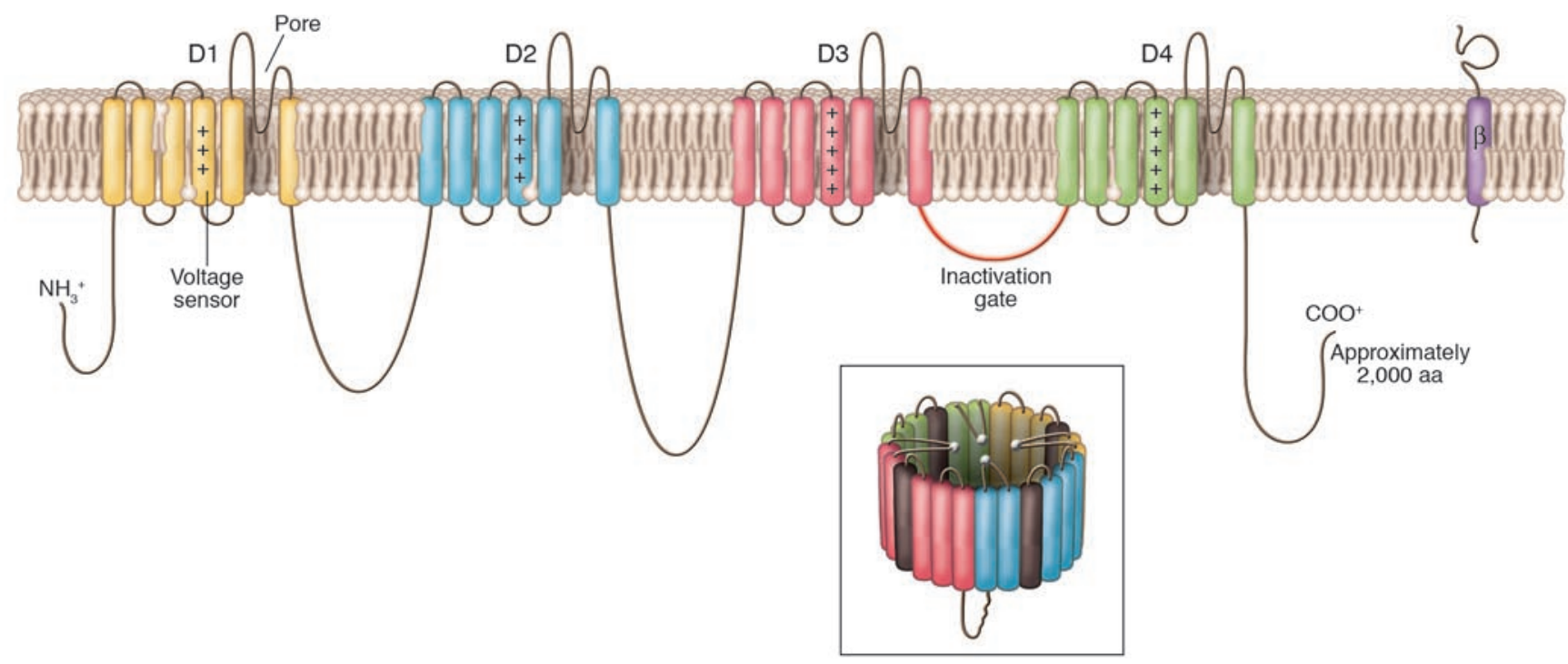

Figure 1

The sodium channel $\alpha$ and $\beta$ subunits are transmembrane proteins. The 4 homologous domains of the $\alpha$ subunit are represented in different colors. The transmembrane segments associate in the membrane to form an $\mathrm{Na}^{+}$-permeable pore lined by the re-entrant S5-S6 pore-loop segments (inset).

\section{Recurrent mutations in SCN1A}

Approximately $25 \%$ of reported mutations in SCN1A have occurred independently more than once $(27,28)$. Examination of the altered nucleotides at the sites of recurrent mutation revealed 2 frequent mechanisms. Fifteen mutations resulted from deamination of ${ }^{\mathrm{m}} \mathrm{CpG}$ dinucleotides, including $5 \mathrm{CpG}$ located in an arginine codon of the CGA class. Deamination converts this codon to UGA, a nonsense codon, resulting in an R-to-X mutation at the amino acid level. Two tetranucleotide direct repeats in SCN1A have been the sites of frameshift mutations due to deletion of 1 copy of the repeat. The likely mechanism is slipped-strand mispairing. The frameshift mutations are at amino acid residues K1846 and L1670.

\section{Rare mutations of SCN2A}

Although SCN1A and SCN2A are closely related genes, similar in size and exon organization, only a few epilepsy mutations have been detected in SCN2A (Figure 2D) (29-32). The difference may be partly accounted for by the likelihood that many more patients have been screened for SCN1A mutations. Most missense mutations of SCN2A were found in patients with benign familial neonatal-infantile seizures (OMIM 607745), mild syndromes that present within the first year of life but do not progress to adult epilepsy (Table 2). One truncation mutation of SCN2A has been identified in a patient with intractable epilepsy resembling SMEI (30).

\section{Haploinsufficiency of SCN1A}

A small proportion of human genes exhibit haploinsufficien$c y$, meaning that abnormal function results from quantitative reduction of gene expression to $50 \%$ of normal levels. Among the epilepsy mutations, the most severe phenotypes are found in individuals who are heterozygous for loss-of-function mutations of SCN1A. This is indicated by the high proportion of truncation mutations among SMEI patients, and the lack of truncation mutations in patients with GEFS+, which is milder (Figure 2, A and B). In SMEI, the clinical features of early onset, seizure severity, and progressive mental deterioration do not differ between patients with truncations located near the $\mathrm{N}$ terminus or the $\mathrm{C}$ terminus, which indicates that loss of function is the common cause. Rhodes et al. examined the functional effect of 5 missense mutations from patients with severe SMEI and found that 2 of those also result in complete loss of channel activity (33).

Unlike SCN1A, SCN2A appears not to exhibit haploinsufficiency. In contrast to the many null mutations of $S C N 1 A$, only a single truncation mutation has been identified in $S C N 2 A$, in a patient with a severe form of epilepsy resembling SMEI (30) (Figure 2D). In this case the truncated protein was reported to have a dominant-negative effect, rather than loss of function (30).

Analysis of mice with knockout alleles supports the conclusion that SCN1A exhibits haploinsufficiency while SCN2A does not. Heterozygotes for the SCN1A knockout allele exhibit spontaneous seizures and reduced lifespan (34), while heterozygotes for the $S C N 2 A$-null allele are viable without visible abnormalities (35). SCN8A-heterozygous-null mice lack visible abnormalities, but

\section{Table 1}

Mammalian voltage-gated sodium channel genes

\begin{tabular}{|c|c|c|c|c|}
\hline Gene & Protein & $\begin{array}{c}\text { Human } \\
\text { chromosome }\end{array}$ & $\begin{array}{c}\text { Major } \\
\text { expression }\end{array}$ & $\begin{array}{c}\text { Minor } \\
\text { expression }\end{array}$ \\
\hline SCN1A & $\mathrm{Na}_{v} 1.1$ & $2 q 24$ & CNS, PNS & Cardiac muscle \\
\hline SCN2A & $\mathrm{Na}_{v} 1.2$ & $2 q 24$ & CNS, PNS & \\
\hline SCN3A & $\mathrm{Na}_{\mathrm{v}} 1.3$ & $2 q 24$ & CNS, PNS & \\
\hline SCN4A & $\mathrm{Na}_{\mathrm{v}} 1.4$ & $17 q 23$ & Skeletal muscle & \\
\hline SCN5A & $\mathrm{Na}_{v} 1.5$ & $3 p 21$ & Cardiac muscle & $\begin{array}{c}\text { Skeletal muscle, } \\
\text { brain }\end{array}$ \\
\hline SCN8A & $\mathrm{Na}_{v} 1.6$ & $12 q 13$ & CNS, PNS & Cardiac muscle \\
\hline SCN9A & $\mathrm{Na}_{v} 1.7$ & $2 q 24$ & PNS & \\
\hline SCN10A & $\mathrm{Na}_{v} 1.8$ & $3 p 21$ & PNS & \\
\hline SCN11A & $\mathrm{Na}_{v} 1.9$ & $3 p 21$ & PNS & \\
\hline
\end{tabular}

PNS, peripheral nervous system. 
A

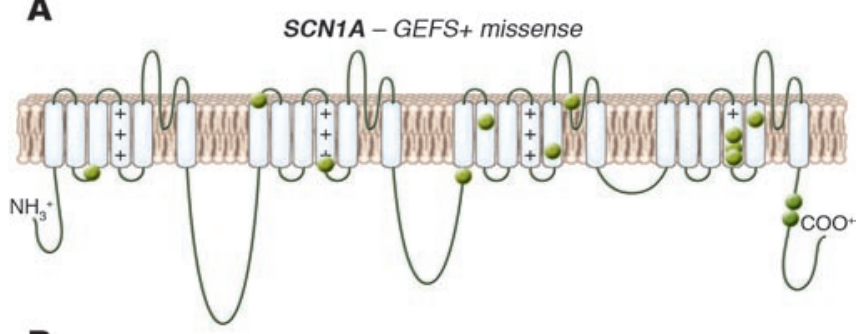

B

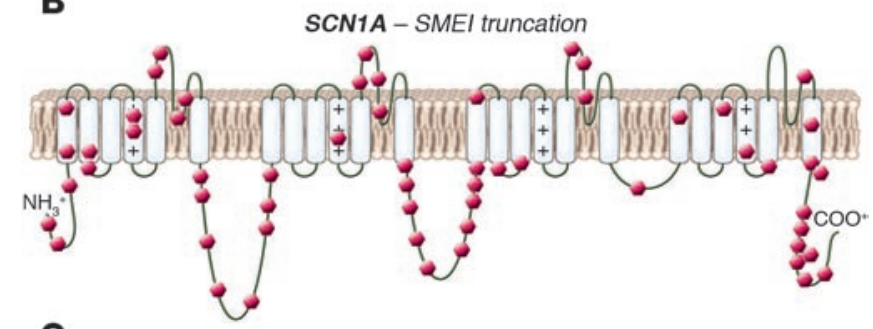

C

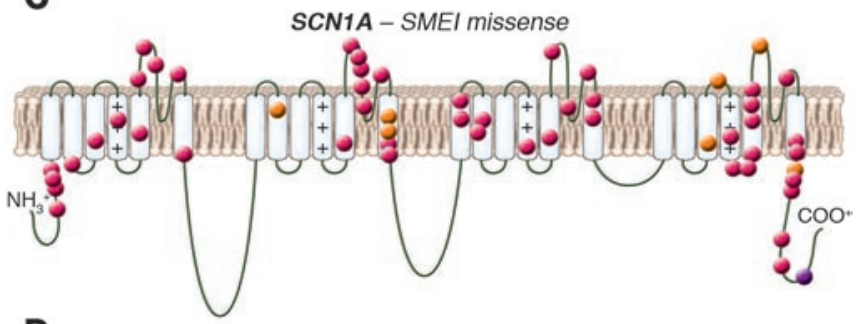

D

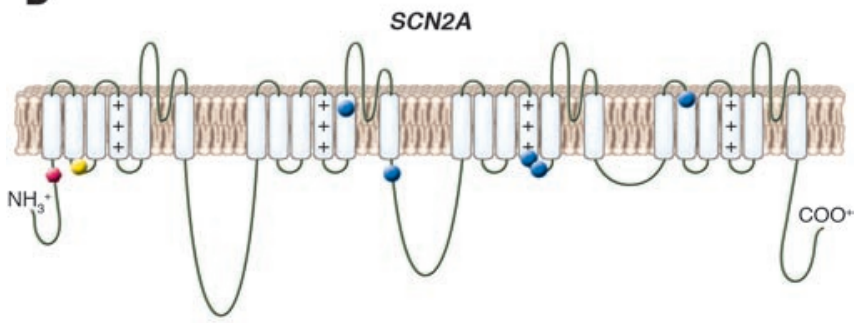

subtle deficiencies have been detected in tests of learning and anxiety (36). The $\beta 1$ and $\beta 2$ genes do not exhibit haploinsufficiency in mice heterozygous for targeted knockout alleles $(37,38)$.

\section{Functional effects of SCN1A missense mutations}

Twenty SCN1A missense mutations have been evaluated in functional assays $(23,24,33,39-46)$. Functional analysis is complicated by the difficulty of cloning neuronal sodium channel cDNAs, which are uniquely unstable during propagation in bacterial cultures (unlike the muscle sodium channel cDNAs or the calcium and potassium channel cDNAs). Functional studies in the Xenopus oocyte system and in transfected mammalian cells do not always agree, and there is little experimental basis for extrapolation to in vivo effects. Nonetheless, some interesting patterns have emerged relating altered channel function to the neuronal hyperexcitability that is thought to underlie seizure disorders.

One common functional abnormality is impaired channel inactivation leading to increased persistent current. Normally, the voltage-gated sodium channels open rapidly in response to altered membrane potential and inactivate rapidly, declining to $1 \%$ of maximal current within a few milliseconds (Figure 3A). In 3 different GEFS+ mutations $(14,19)$, persistent current was increased to $2-5 \%$ of peak current (39) (Figure $3 \mathrm{~A}$ ). In the context of the neu-

\section{Figure 2}

More than 150 mutations in the sodium channel protein have been identified in patients with GEFS+ and SMEI. (A) Missense mutations of SCN1A identified in families with GEFS+ $(14,16,17,19,21-25)$. (B) Truncation mutations of SCN1A identified in SMEl patients $(18,26$, 27, 44, 47, 50, 53, 54, 79-84). (C) Missense mutations of SCN1A in patients with SMEI (red), intractable childhood epilepsy with generalized tonic-clonic seizures (ICEGTC) (orange), and infantile spasms (purple) $(18,26,27,44,47,50,53,79-84)$. (D) Mutations of SCN2A in patients with benign familial neonatal-infantile seizures (BFNIS) (blue), GEFS+ (yellow), and SMEI (red) (29-32).

ron, this persistent current is thought to reduce the depolarization threshold required for firing, resulting directly in hyperexcitability. Another common mechanism is demonstrated by the GEFS+ mutation $\mathrm{D} 188 \mathrm{~V}$, which spends less time in the inactivated state than the wild-type channel (Figure 3B). The result is greater availability of channels for opening in response to depolarization, another route to hyperexcitability. The altered biophysical properties of representative mutant channels are summarized in Table 3.

A unique biochemical mechanism was observed for the missense mutation D1866Y, located in the C-terminal domain of SCN1A, in a family with GEFS+ (23). In the Xenopus oocyte system, the mutant channel exhibited a depolarized shift in voltage dependence of fast inactivation; the effect was tenfold greater in the presence of the $\beta 1$ subunit (Figure $3 \mathrm{C}$ ). Modeling with the program NEURON (http://www.neuron.yale.edu/neuron/) indicated that this shift is sufficient to produce neuronal hyperexcitability. Because the difference between the wild-type and mutant channels was increased by the presence of the $\beta 1$ subunit, the effect of the mutation on subunit interaction was tested. Yeast 2-hybrid screen and coimmunoprecipitation demonstrated direct interaction between the C-terminal cytoplasmic domains of the $\alpha$ and $\beta$ subunits, which was impaired by the D1866Y mutation (23). The D1866Y mutation thus defines an intracellular interaction domain that appears to be required, in combination with the extracellular interaction domain (23), to form the stable $\alpha / \beta$ complex. Since mutations in either SCN1A or $\beta 1$ can result in GEFS+, it is not surprising that impaired interaction between the 2 subunits could also cause the disease.

The GEFS+ mutation R1648H has been examined in 3 expression systems with different outcomes. When the mutation was introduced into the rat SCN1A cDNA and examined in the Xenopus

\section{Table 2}

Sodium channel mutations in patients with various types of epilepsy

\begin{tabular}{|c|c|c|c|}
\hline & SCN1A $\mathrm{Na}_{v} 1.1$ & SCN2A $\mathrm{Na}_{v} 1.2$ & SCN1B $\beta 1$ \\
\hline $\begin{array}{l}\text { SMEI } \\
\quad \text { (at least 90\% } \\
\text { sporadic) }\end{array}$ & 150 & 1 & - \\
\hline GEFS+ & 13 & 1 & 2 \\
\hline ICEGTC & 7 & - & - \\
\hline Infantile spasms & 1 & - & - \\
\hline $\begin{array}{l}\text { Benign familial } \\
\text { neonatal-infantile }\end{array}$ & - & 6 & - \\
\hline
\end{tabular}

ICEGTC, intractable childhood epilepsy with generalized tonic-clonic seizures. 
A
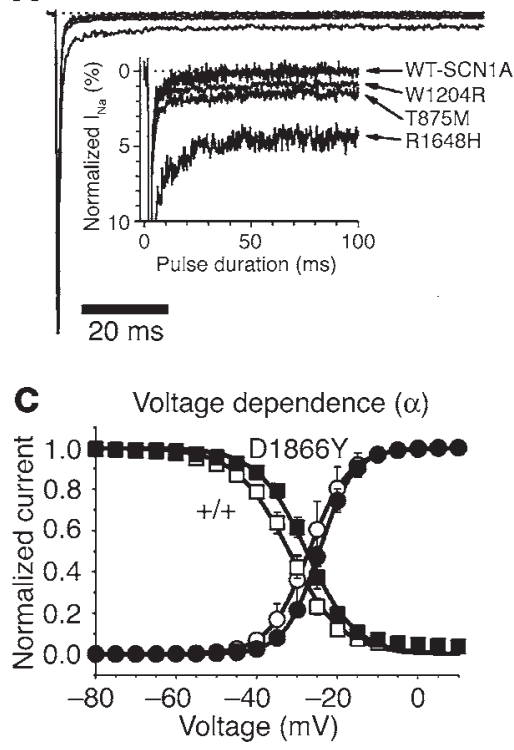

B
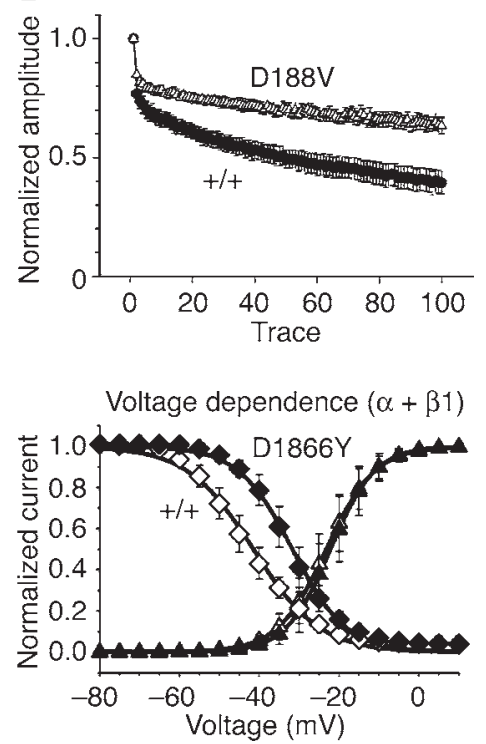

Figure 3

The effects of GEFS+ mutations on SCN1A channel properties have been studied in the Xenopus oocyte system and in transfected mammalian cells. (A) Whole-cell recordings from HEK tsA201 cells transfected with the indicated mutant SCN1A cDNAs demonstrate increased persistent current from the mutant channels (39). (B) Mean normalized amplitudes of sodium currents elicited by $80-\mathrm{Hz}$ pulse trains in HEK cells expressing SCN2A cDNA containing the GEFS+ mutation D188V demonstrate reduced cumulative inactivation of the mutant channel during high-frequency trains of channel activation (40). (C) Voltage-dependent gating of SCN1A cDNA constructs in Xenopus oocytes expressed in the absence (left) or presence (right) of the $\beta 1$ cDNA (85). See text for discussion. oocyte expression system, accelerated recovery from inactivation was observed (45). In the human cDNA in transfected mammalian cells, persistent current was the major effect (Figure 3A) (39). Alekov et al. introduced $\mathrm{R} 1648 \mathrm{H}$ into the SCN4A cDNA and expressed the clone in mammalian HEK tsA201 cells. In this context, they observed slowed inactivation and accelerated recovery from inactivation, leading to increased channel availability, but no persistent current (42). A second substitution at the same residue, R1648C, was identified in a patient with SMEI (47). In transfected cells, persistent current was generated by R1648C at a level indistinguishable from that of the mutation $\mathrm{R} 1648 \mathrm{H}$ that causes GEFS+ (33). Thus, the heterologous expression systems are not able to distinguish between missense mutations that lead to mild disease in vivo and those that lead to severe disease.

The data indicate that seizures can result from increased SCN1A channel activity, as in the missense mutations described above, and from reduced activity, as in the truncation mutations. Neuronal firing patterns appear to be extremely sensitive to subtle changes in sodium channel function. In the future, the most physiologically relevant data are likely to be obtained from measurements of neuronal currents in knock-in mouse models carrying human mutations.
We investigated the in vivo effect of an SCN2A mutation with impaired inactivation in the Q54 transgenic mouse (48). Analysis of the mutation SCN2A GAL879-881QQQ in the Xenopus oocyte system revealed an increase in persistent current and in the percentage of current that inactivated with a slow time constant (Figure 4, $B$ and C) (49). When the mutant cDNA was expressed in transgenic mice under the control of the neuron-specific enolase promoter, the mice exhibited progressive seizures of hippocampal origin accompanied by loss of neurons called hippocampal sclerosis (Figure 4, A and E). Persistent sodium current was detected in recordings from CA1 neurons of the transgenic mice (Figure 4D), demonstrating agreement between the Xenopus assay and the in vivo effect. The phenotype of the Q54 mice most closely resembles human mesial temporal lobe epilepsy.

\section{Toward mutation-specific therapy}

Selecting the appropriate antiepileptic drug for newly diagnosed epilepsy patients is a difficult process, and many drugs have adverse side effects. An important goal of mutation characterization is the development of individualized treatments tailored to each patient mutation. It is already clear that sodium channel blockers are con-

\section{Table 3}

A variety of functional abnormalities in mutant alleles of SCN1A encoding the sodium channel $\mathrm{Na}_{v} 1.1$

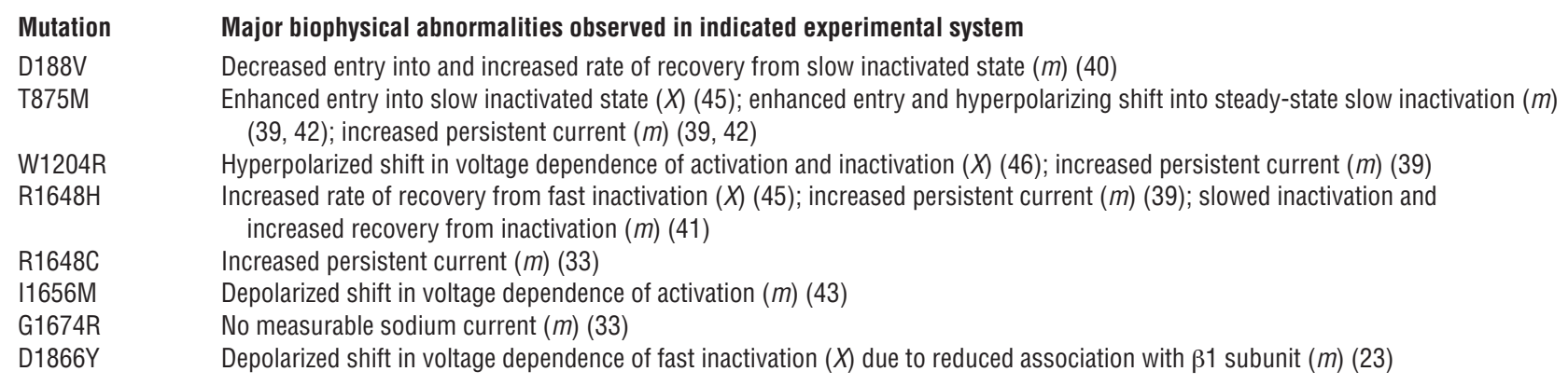




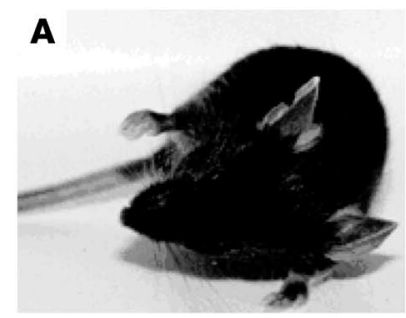

B

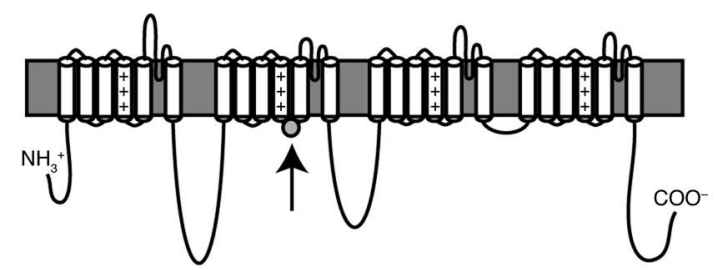

C

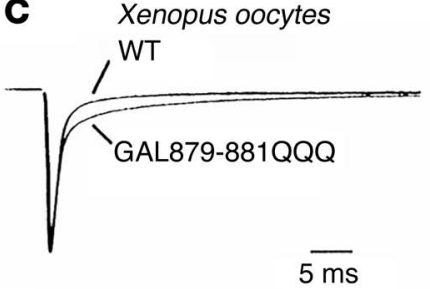

E

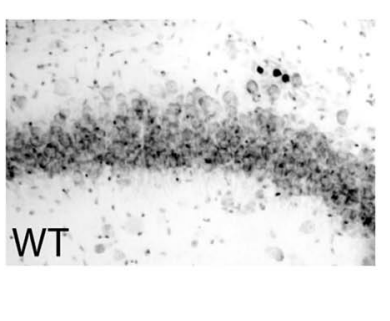

D CA1 neurons
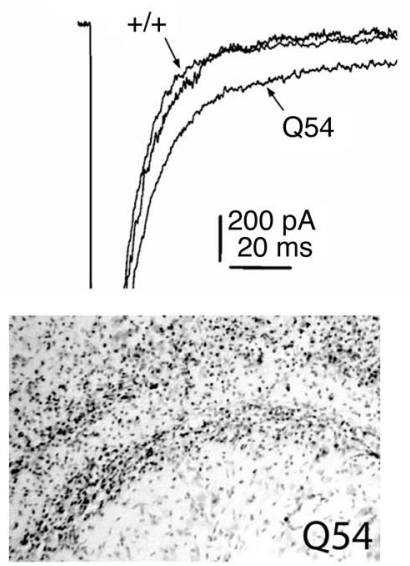

Figure 4

An SCN2A mutation with persistent current causes seizures in the Q54 transgenic mouse. (A) Focal motor seizure in a Q54 mouse. (B) The GAL879-881QQQ mutation is located in the D2S4-S5 linker. (C) The mutant channel generates persistent current in Xenopus oocytes. (D) Whole-cell sodium currents recorded from CA1 hippocampal neurons from presymptomatic Q54 mice demonstrate increased persistent current. (E) Nissl-stained sections of hippocampus area CA3 reveal extensive neuronal cell loss in a Q54 mouse compared with a wild-type littermate. Adapted with permission from Neuroscience (48).

traindicated for patients with SMEI who are heterozygous for null alleles of SCN1A, since further reduction of the level of functional channel worsens their condition. In the case of mutations causing persistent current, open-state blockers may be appropriate. Pharmacological analysis of mutant SCN5A channels in LQT-3 provides a model for development of mutation-targeted therapy for a sodium channel defect $(50,51)$. Genetic variation in drugmetabolizing enzymes may also be predictive of pharmacological effectiveness of antiepileptic drugs (52).

\section{Variable expressivity and incomplete penetrance}

Variable expressivity, differences in clinical severity among individuals carrying the same mutation, is a striking feature of both GEFS+ and SMEI. For example, in the GEFS+ family carrying the mutation T875M, approximately half of the heterozygotes experienced febrile seizures in childhood only, while the other half had a variety of seizure types as adults (14). As another example, although most SMEI mutations arise de novo in affected individuals, a few patients are known to have inherited the SCN1A mutation from a mildly affected parent $(53,54)$. Several factors may be considered as contributing to differences between individuals carrying the same sodium channel mutation. There is intrinsic stochastic variability in developmental processes even between inbred animals homozygous throughout the genome, as demonstrated by the variable phenotypes of inbred mice with certain mutant genotypes. These processes may be especially important during development of neuronal connectivity in the CNS. Second, it has been suggested that the accumulation of somatic mutations during the lifetime of an individual may contribute to the development of neurological disease, and especially focal disorders such as epilepsy (55). Third, environmental insults such as trauma may exacerbate the phenotype of individuals carrying a mild mutation. Fourth, multiple differences in genetic background, or modifier genes, are segregating in families, and the combined effect of multiple susceptibility factors may result in greater clinical severity in some individuals.

The influence of modifier genes is amenable to analysis using genetic differences between inbred strains of mice as models. Modifier genes that influence susceptibility to spontaneous seizures (56), kainate-induced seizures (57), electroconvulsive shock (58), and pentylenetetrazol-induced seizures (59) have been mapped to specific mouse chromosomes. Recently, we mapped 2 modifier loci, Moe1 (modifier of epilepsy) and Moe2, that influence clinical severity of epilepsy due to a sodium channel mutation in $\operatorname{Scn} 2 a^{Q 54}$ mice (60). With current genomic technology, it is feasible to clone modifier genes and evaluate their role in human disease. For example, a gene that modifies the severity of an SCN8A-induced movement disorder was recently cloned and identified as a putative RNA splicing factor (61).

\section{SCN8A and movement disorders}

Mutations of Scnsa in the mouse result in movement disorders such as ataxia, dystonia, and tremor (62). Conditional inactivation of Scnsa in cerebellar neurons results in a milder ataxia $(63,64)$. Screening of 150 patients with sporadic or inherited ataxia identified 1 protein truncation mutation that is likely to cause loss of function of SCN8A (36). Further screening will be worthwhile to determine the prevalence of $S C N 8 A$ mutations in patients with inherited and sporadic movement disorders.

\section{SCN9A and inherited pain}

$S C N 9 A$ encodes the sodium channel $\mathrm{Na}_{v} 1.7$, which is expressed in peripheral sensory and sympathetic neurons and is localized in the nerve terminals of sensory neurons (65). The missense mutation $\mathrm{L} 858 \mathrm{H}$, in a transmembrane segment, was identified in a Chinese kindred with autosomal dominantly inherited primary erythermalgia (OMIM 113020), a disorder characterized by intermittent pain, redness, heat, and swelling in the extremities (66). A second mutation 
Table 4

Mouse models of neurological disorders with sodium channel mutations

\begin{tabular}{|c|c|c|c|c|}
\hline Gene & Channel & Mutation & Disorder & Reference \\
\hline SCN1A & $\mathrm{Na}_{\mathrm{v}} 1.1$ & Targeted null & $\begin{array}{l}\text { Seizures in heterozygote; } \\
\text { homozygous lethal at P16 }\end{array}$ & (34) \\
\hline \multirow[t]{2}{*}{ SCN2A } & \multirow[t]{2}{*}{$\mathrm{Na}_{\mathrm{v}} 1.2$} & Targeted null & $\begin{array}{l}\text { No abnormalities in heterozygote; } \\
\text { homozygous lethal at P1 }\end{array}$ & $(35)$ \\
\hline & & $\begin{array}{l}\text { Q54 transgene } \\
(\mathrm{GAL} \rightarrow Q Q Q)\end{array}$ & $\begin{array}{l}\text { Dominantly inherited temporal } \\
\text { lobe epilepsy }\end{array}$ & $(48)$ \\
\hline \multirow[t]{5}{*}{ SCN8A } & \multirow[t]{5}{*}{$\mathrm{Na}_{v} 1.6$} & Spontaneous null & Paralysis, lethal & (62) \\
\hline & & Null heterozygote & Learning deficits & (36) \\
\hline & & A1071T & Ataxia, tremor & (86) \\
\hline & & Hypomorph (medJ) & Dystonia & (87) \\
\hline & & Conditional null & CRE-dependent lethal & (64) \\
\hline \multirow[t]{2}{*}{ SCN9A } & \multirow[t]{2}{*}{$\mathrm{Na}_{\mathrm{v}} 1.7$} & Targeted null & Lethal & $(70)$ \\
\hline & & Conditional null & Pain resistant & (70) \\
\hline SCN10A & $\mathrm{Na}_{v} 1.8$ & Targeted null & Pain resistant & (88) \\
\hline SCN1B & $\beta 1$ & Targeted null & $\begin{array}{l}\text { Seizures in heterozygote; } \\
\text { lethal seizures in homozygote }\end{array}$ & (38) \\
\hline SCN2B & $\beta 2$ & Targeted null & $\begin{array}{l}\text { Heterozygote normal; } \\
\text { homozygote susceptible to seizures }\end{array}$ & $(37)$ \\
\hline
\end{tabular}

sidered candidates for a causal role in autism. The missense mutation R1902C, located within a calmodulin-binding domain of SCN2A, was subsequently found to confer a calcium-dependent conformational change on the complex of calmodulin with the $\mathrm{C}$ terminus of $\mathrm{Na}_{\mathrm{v}} 1.2$ (72). In this context it is interesting that mutations of the voltage-gated calcium channel $\mathrm{Ca}_{v} 1.2$ were recently identified in families with Timothy syndrome, a multiorgan disorder with autism as a feature (73).

Preliminary indication that sodium channel variants may influence cognitive function comes from heterozygotes for a null allele of $S C N 8 A$, who exhibit a variety of cognitive defects (36). Impaired learning was also observed in heterozygous-null mice (36). An influence of SCN8A on attempted suicide was indicated by preferential transmission of 1 allele of a single-nucleotide polymorphism

in a nearby residue, I848T, was found in a sporadic case of erythermalgia. Functional analysis detected a hyperpolarizing shift in activation and slowed deactivation in both mutants, as well as increased current amplitude in response to slow, small depolarizations, consistent with predicted hyperactivity at the cellular level (67). A third mutation, F1449V, close to D3S6, lowers the current threshold required for action potential generation and repetitive firing (68).

The syndrome familial rectal pain (OMIM 167400) is characterized by brief episodes of intense pain of the submandibular, ocular, and rectal areas with flushing of the surrounding skin. The pain is responsive to the sodium channel blocker carbamazepine. Missense mutations in SCN9A have recently been identified in several families with this disorder (69).

Conditional inactivation of Scn9a in sensory neurons of the mouse resulted in increased threshold for mechanical, thermal, and inflammatory pain (70). The association of SCN9A mutations with inherited pain syndromes, and the pain resistance of the knockout mouse, indicate that specific inhibitors of this sodium channel would be valuable for the treatment of chronic pain. Thus far, the evolutionary conservation of the mammalian $\alpha$ subunit genes has made it difficult to develop inhibitors with specificity for a single channel.

\section{Sodium channels and psychiatric disease?}

There is a biological continuum between neurological diseases such as epilepsy and ataxia, and disorders that have been traditionally classified as psychiatric. Because of the essential role of sodium channels in propagating neuronal signals throughout the CNS, and the sensitivity of neuronal firing patterns to subtle mutations in sodium channels, it seems that sodium channel mutations could directly affect emotional and cognitive function. To test this possibility, we screened for mutations in SCN1A, SCN2A, and $S C N 3 A$ in multiplex families with autism, a psychiatric disorder with high heritability (71). One splice branchpoint and 5 missense mutations were observed among 117 families; these may be con- in intron 21 (74). Autistic features have also been reported in patients with SMEI. Clearly, much work will be required to follow up on these intriguing observations regarding the potential influence of sodium channel and other neuronal ion channel variants on cognitive and psychiatric traits.

\section{Mice with neuronal sodium channel mutations}

The development of mouse models is keeping pace with the identification of human mutations, and several lines are available for analysis of disease mechanisms. The features and availability are summarized in Table 4. Four lines with various types of seizures are already available, the Q54 transgenic line with a missense mutation of $S C N 2 A$ and the knockout lines for $S C N 1 A, S C N 1 B(\beta 1)$, and $S C N 2 B$ $(\beta 2)$. Generation of tissue-specific and inducible knock-in mice carrying specific epilepsy-related sodium channel mutations is in progress in several laboratories. Electrophysiological recordings of neurons from knock-in mice are likely to provide the most reliable information for understanding the relationship between specific mutations and the severity of disease. Because of the effort involved in generating these models, only a small number of selected mutations can be analyzed in vivo. Recordings from mice with SCN8A mutations have revealed different effects on sodium currents in different types of neurons (75-77). In Purkinje cells, for example, SCN8A deficiency reduces bursting activity and resurgent current (77), while in cortical pyramidal neurons the major effect is reduced persistent sodium current (76). Analysis of SCN1A-null homozygotes revealed reduced current density in interneurons but not in excitatory neurons of the hippocampus (34). In addition to elucidating the pathophysiology of epileptogenesis, mouse models will permit evaluation of therapies directed toward specific classes of sodium channel mutations.

\section{Persistent issues: sodium channels and disease}

The identification of nearly 200 sodium channel mutations in patients with epilepsy raises several issues for future research. The relatively subtle functional effects of some of the epilepsy mutations 
suggest that the nervous system is intolerant of even minor variation in the properties of these channel proteins, which have a direct role in neuronal firing. The large number of de novo mutations in the sporadic disorder SMEI suggests that mutation should be considered as a possible cause of neurological disease even in the absence of family history. A de novo mutation of SCN9A was identified in a sporadic case of erythermalgia. Development of genotyping technology has made it easier to screen for mutations, and a commercial sequencing test for SCN1A mutations was recently introduced. However, the cost of several thousand dollars per test, as well as ethical and practical concerns about the implications of mutation detection, may slow the application of genetic testing for neurological disease.

Evaluation of coding variants detected in genetic screens poses significant challenges. Which missense mutations cause disease and which are neutral? Large numbers of unaffected controls (approximately 1,000) must be tested to provide statistically significant evidence that a variant discovered in a patient is not also found in unaffected individuals. Functional assays can be helpful in recognizing disease mutations, but the difficulty of generating expression constructs and interpreting assays in exogenous expression systems makes these tests problematic for clinical applications.

Does quantitative variation in sodium channel abundance due to mutation in noncoding regulatory sequences contribute to neurological disease? The haploinsufficiency of SCN1A revealed by the loss-of-function coding mutations suggests that reduced expression itself may be sufficient to cause disease. Screening for variation in expression requires access to neuronal RNA samples. Recent studies using cDNA microarray hybridization or allele-specific primer extension reactions suggest that as many as $20 \%$ of human genes may vary in expression levels among individuals (78). This interesting area remains essentially unexplored.

If we could identify variants in modifier genes underlying the variable expressivity of sodium channel mutations, perhaps using animal models carrying human mutations, new targets for treatment intervention might result. The growing role of sodium channel mutations in neurological disease provides increased incentive for developing new and more specific sodium channel inhibitors. We anticipate that large-scale sequencing of ion channel genes from patients with epilepsy and other neurological disorders will be carried out within the next few years, with major impact on our understanding of the issues discussed here.

\section{Acknowledgments}

This work was supported by NIH research grants R01 NS34509 (to M.H. Meisler) and R21 NS046315 (to J.A. Kearney). We thank Jorgen de Haan, Valerie Drews, Lori Isom, and Christoph Lossin for helpful discussions.

Address correspondence to: Miriam H. Meisler, Department of Human Genetics, University of Michigan Medical School, 4909 Buhl Box 0618, Ann Arbor, Michigan 48109-0618, USA. Phone: (734) 763-5546; Fax: (734) 763-9691; E-mail: meislerm@umich.edu.
1. Jiang, Y., et al. 2003. X-ray structure of a voltagedependent $\mathrm{K}+$ channel. Nature. 423:33-41.

2. Jiang, Y., Ruta, V., Chen, J., Lee, A., and MacKinnon, R. 2003. The principle of gating charge movement in a voltage-dependent $\mathrm{K}+$ channel. Nature. 423:42-48.

3. Isom, L.L., De Jongh, K.S., and Catterall, W.A. 1994. Auxiliary subunits of voltage-gated ion channels. Neuron. 12:1183-1194.

4. Yu, F.H., et al. 2003. Sodium channel $\beta 4$, a new disulfide-linked auxiliary subunit with similarity to $\beta 2$. J. Neurosci. 23:7577-7585.

5. Plummer, N.W., and Meisler, M.H. 1999. Evolution and diversity of mammalian sodium channel genes. Genomics. 57:323-331.

6. Goldin, A.L. 2001. Resurgence of sodium channel research. Annu. Rev. Physiol. 63:871-894.

7. Scheffer, I., and Berkovic, S.F. 1997. Generalized epilepsy with febrile seizures plus. A genetic disorder with heterogeneous clinical phenotypes. Brain. 120:479-490.

8. Singh, R., Scheffer, I.E., Crossland, K., and Berkovic, S.F. 1999. Generalized epilepsy with febrile seizures plus: a common childhood-onset genetic epilepsy syndrome. Ann. Neurol. 45:75-81.

9. Wallace, R., et al. 1998. Febrile seizures and generalized epilepsy associated with a mutation in the Na+-channel beta1 subunit gene SCN1B. Nat. Genet. 19:366-370.

10. Meadows, L.S., et al. 2002. Functional and biochemical analysis of a sodium channel beta 1 subunit mutation responsible for generalized epilepsy with febrile seizures plus type 1. J. Neurosci. 22:10699-10709

11. Audenaert, D., et al. 2003. A deletion in SCN1B is associated with febrile seizures and early-onset absence epilepsy. Neurology. 61:854-856.

12. Baulac, S., et al. 1999. A second locus for familial generalized epilepsy with febrile seizures plus maps to chromosome 2q21-q33. Am. J. Hum. Genet. 65:1078-1085.

13. Moulard, B., et al. 1999. Identification of a new locus for generalized epilepsy with febrile seizures plus (GEFS+) on chromosome 2q24-q33. Am. J. Hum. Genet. 65:1396-1400.

14. Escayg, A., et al. 2000. Mutations of SCN1A, encoding a neuronal sodium channel, in two families with GEFS+2. Nat. Genet. 24:343-345.

15. Bonanni, P., et al. 2004. Generalized epilepsy with febrile seizures plus (GEFS+): clinical spectrum in seven Italian families unrelated to SCN1A, SCN1B, and GABRG2 gene mutations. Epilepsia. 45:149-158.

16. Abou-Khalil, B., et al. 2001. Partial and generalized epilepsy with febrile seizures plus and a novel SCN1A mutation. Neurology. 57:2265-2272.

17. Annesi, G., et al. 2003. Two novel SCN1A missense mutations in generalized epilepsy with febrile seizures plus. Epilepsia. 44:1257-1258.

18. Ceulemans, B.P., Claes, L.R., and Lagae, L.G. 2004. Clinical correlations of mutations in the SCN1A gene: from febrile seizures to severe myoclonic epilepsy in infancy. Pediatr. Neurol. 30:236-243.

19. Escayg, A., et al. 2001. A novel SCN1A mutation associated with generalized epilepsy with febrile seizures plus - and prevalence of variants in patients with epilepsy. Am. J. Hum. Genet. 68:866-873.

20. Gerard, F., Pereira, S., Robaglia-Schlupp, A., Genton, P., and Szepetowski, P. 2002. Clinical and genetic analysis of a new multigenerational pedigree with GEFS+ (generalized epilepsy with febrile seizures plus). Epilepsia. 43:581-586.

21. Ito, M., et al. 2002. Autosomal dominant epilepsy with febrile seizures plus with missense mutations of the $\left(\mathrm{Na}^{+}\right)$-channel alpha 1 subunit gene, SCN1A. Epilepsy Res. 48:15-23.

22. Lerche, H., et al. 2001. Generalized epilepsy with febrile seizures plus: further heterogeneity in a large family. Neurology. 57:1191-1198.

23. Spampanato, J., et al. 2004. A novel epilepsy mutation in the sodium channel SCN1A identifies a cytoplasmic domain for beta subunit interaction. J. Neurosci. 24:10022-10034.

24. Sugawara, T., et al. 2001. Nav1.1 mutations cause febrile seizures associated with afebrile partial seizures. Neurology. 57:703-705.

25. Wallace, R.H., et al. 2001. Neuronal sodium-channel alpha1-subunit mutations in generalized epilepsy with febrile seizures plus. Am. J. Hum. Genet. 68:859-865.

26. Claes, L., et al. 2001. De novo mutations in the sodium-channel gene SCN1A cause severe myoclonic epilepsy of infancy. Am. J. Hum. Genet. 68:1327-1332

27. Wallace, R.H., et al. 2003. Sodium channel alpha1subunit mutations in severe myoclonic epilepsy of infancy and infantile spasms. Neurology. 61:765-769.

28. Fukuma, G., et al. 2004. Mutations of neuronal voltage-gated $\mathrm{Na}+$ channel alpha1 subunit gene SCN1A in core severe myoclonic epilepsy in infancy (SMEI) and in borderline SMEI (SMEB). Epilepsia. 45:140-148.

29. Sugawara, T., et al. 2001. A missense mutation of the $\mathrm{Na}+$ channel alpha II subunit gene $\mathrm{Na}(\mathrm{v}) 1.2$ in a patient with febrile and afebrile seizures causes channel dysfunction. Proc. Natl. Acad. Sci. U. S. A. 98:6381-6389.

30. Kamiya, K., et al. 2004. A nonsense mutation of the sodium channel gene SCN2A in a patient with intractable epilepsy and mental decline. J. Neurosci. 24:2690-2698.

31. Heron, S.E., et al. 2002. Sodium-channel defects in benign familial neonatal-infantile seizures. Lancet. 360:851-852.

32. Berkovic, S.F., et al. 2004. Benign familial neonatalinfantile seizures: characterization of a new sodium channelopathy. Ann. Neurol. 55:550-557.

33. Rhodes, T.H., Lossin, C., Vanoye, C.G., Wang, D.W., and George, A.L., Jr. 2004. Noninactivating voltage-gated sodium channels in severe myoclonic epilepsy of infancy. Proc. Natl. Acad. Sci. U. S. A. 101:11147-11152.

34. Yu, F.H., et al. 2004. Deletion of the Nav1.1 channel: a mouse model for severe myoclonic epilepsy of infancy. Program no. 479.5 presented at the 34 th 
Annual Society for Neuroscience Meeting. October 25. San Diego, California, USA. http://sfn.scholarone.com/itin2004/index.html.

35. Planells-Cases, R., et al. 2000. Neuronal death and perinatal lethality in voltage-gated sodium channel alpha(II)-deficient mice. Biophys. J. 78:2878-2891.

36. Trudeau, M.M., Dalton, J.D., Day, J.W., Ranum, L.P.W., and Meisler, M.H. 2004. Heterozygosity for a truncation allele of sodium channel SCN8A in a family with ataxia and cognitive impairment. Program no. 205 presented at the 54th Annual Meeting of the American Society of Human Genetics. October 30. Bethesda, Maryland, USA. http://www. ashg.org/genetics/ashg04s/index.shtml.

37. Chen, C., et al. 2002. Reduced sodium channel density, altered voltage dependence of inactivation, and increased susceptibility to seizures in mice lacking sodium channel beta 2-subunits. Proc. Natl. Acad. Sci. U. S. A. 99:17072-17077.

38. Chen, C., et al. 2004. Mice lacking sodium channel beta1 subunits display defects in neuronal excitability, sodium channel expression, and nodal architecture. J. Neurosci. 24:4030-4042.

39. Lossin, C., Wang, D.W., Rhodes, T.H., Vanoye, C.G., and George, A.L., Jr. 2002. Molecular basis of an inherited epilepsy. Neuron. 34:877-884.

40. Cossette, P., et al. 2003. Functional characterization of the D188V mutation in neuronal voltagegated sodium channel causing generalized epilepsy with febrile seizures plus (GEFS). Epilepsy Res. 53:107-117.

41. Alekov, A., Rahman, M.M., Mitrovic, N., LehmannHorn, F., and Lerche, H. 2000. A sodium channel mutation causing epilepsy in man exhibits subtle defects in fast inactivation and activation in vitro. J. Physiol. 529:533-539.

42. Alekov, A.K., Rahman, M.M., Mitrovic, N., LehmannHorn, F., and Lerche, H. 2001. Enhanced inactivation and acceleration of activation of the sodium channel associated with epilepsy in man. Eur. J. Neurosci. 13:2171-2176

43. Lossin, C., et al. 2003. Epilepsy-associated dysfunction in the voltage-gated neuronal sodium channel SCN1A. J. Neurosci. 23:11289-11295.

44. Sugawara, T., et al. 2003. Nav1.1 channels with mutations of severe myoclonic epilepsy in infancy display attenuated currents. Epilepsy Res. 54:201-207.

45. Spampanato, J., Escayg, A., Meisler, M.H., and Goldin, A.L. 2001. Functional effects of two voltagegated sodium channel mutations that cause generalized epilepsy with febrile seizures plus type 2 . J. Neurosci. 21:7481-7490.

46. Spampanato, J., Escayg, A., Meisler, M.H., and Goldin, A.L. 2003. Generalized epilepsy with febrile seizures plus type 2 mutation W1204R alters voltage-dependent gating of Nav1.1 sodium channels. Neuroscience. 116:37-48.

47. Ohmori, I., Ouchida, M., Ohtsuka, Y., Oka, E., and Shimizu, K. 2002. Significant correlation of the SCN1A mutations and severe myoclonic epilepsy in infancy. Biochem. Biophys. Res. Commun. 295:17-23.

48. Kearney, J.A., et al. 2001. A gain-of-function mutation in the sodium channel gene $S \mathrm{cn} 2 \mathrm{a}$ results in seizures and behavioral abnormalities. Neuroscience. 102:307-317.

49. Smith, M.R., and Goldin, A.L. 1997. Interaction between the sodium channel inactivation linker and domain III S4-S5. Biophys. J. 73:1885-1895.

50. Abriel, H., Wehrens, X.H.T., Benhorin, J., Kerem, B., and Kass, R.S. 2000. Molecular pharmacology of the sodium channel mutation D1790G linked to the long-QT syndrome. Circulation. 102:921-925.
51. Benhorin, J., et al. 2000. Effects of flecainide in patients with new SCN5A mutation: mutationspecific therapy for long-QT syndrome? Circulation. 101:1698-1706.

52. Tate, S.K., et al. 2005. Genetic predictors of the maximum doses patients receive during clinical use of the anti-epileptic drugs carbamazepine and phenytoin. Proc. Natl. Acad. Sci. U. S. A. 102:5507-5512.

53. Gennaro, E., et al. 2003. Familial severe myoclonic epilepsy of infancy: truncation of Nav1.1 and genetic heterogeneity. Epileptic Disord. 5:21-25.

54. Fujiwara, T., et al. 2003. Mutations of sodium channel $\alpha$ subunit type 1 (SCN1A) in intractable childhood epilepsies with frequent generalized tonic-clonic seizures. Brain. 126:531-546.

55. Weiss, K.M. 2005. Cryptic causation of human disease: reading between the (germ) lines. Trends Genet. 21:82-88.

56. Legare, M.E., Bartlett, F.S., 2nd, and Frankel, W.N. 2000. A major effect QTL determined by multiple genes in epileptic EL mice. Genome Res. 10:42-48.

57. Schauwecker, P.E., Williams, R.W., and Santos, J.B. 2004. Genetic control of sensitivity to hippocampal cell death induced by kainic acid: a quantitative trait loci analysis. J. Comp. Neurol. 477:96-107.

58. Ferraro, T.N., et al. 2004. Fine mapping of a seizure susceptibility locus on mouse chromosome 1 : nomination of Kenj10 as a causative gene. Mamm. Genome. 15:239-251.

59. Ferraro, T.N., et al. 1999. Mapping loci for pentylenetetrazol-induced seizure susceptibility in mice. J. Neurosci. 19:6733-6739.

60. Bergren, S.K., Chen, S., Galecki, A., and Kearney, J.A. 2005. Genetic modifiers affecting severity of epilepsy caused by mutation of sodium channel Scn2a. Mamm. Genome. In press.

61. Buchner, D.A., Trudeau, M., and Meisler, M.H. 2003. SCNM1, a putative RNA splicing factor that modifies disease severity in mice. Science. 301:967-969.

62. Meisler, M.H., Kearney, J., Escayg, A., MacDonald, B.T., and Sprunger, L.K. 2001. Sodium channels and neurological disease: insights from Scn8a mutations in the mouse. Neuroscientist. 7:136-145.

63. Levin, S.I., and Meisler, M.H. 2004. Conditional inactivation of the voltage-gated sodium channel $\mathrm{Scn} 8 \mathrm{a}\left(\mathrm{Na}_{\mathrm{v}} 1.6\right)$ in cerebellar Purkinje and granule cells. Program no. 397.1 presented at the Annual Society for Neuroscience Meeting. October 23-27. San Diego, California, USA. http://sfn.scholarone. com/itin2004/index.html.

64. Levin, S.I., and Meisler, M.H. 2004. Floxed allele for conditional inactivation of the voltage-gated sodium channel Scn8a (NaV1.6). Genesis. 39:234-239.

65. Wood, J.N., Boorman, J.P., Okuse, K., and Baker, M.D. 2004. Voltage-gated sodium channels and pain pathways. J. Neurobiol. 61:55-71.

66. Yang, Y., et al. 2004. Mutations in SCN9A, encoding a sodium channel alpha subunit, in patients with primary erythermalgia. J. Med. Genet. 41:171-174.

67. Cummins, T.R., Dib-Hajj, S.D., and Waxman, S.G. 2004. Electrophysiological properties of mutant Nav1.7 sodium channels in a painful inherited neuropathy. J. Neurosci. 24:8232-8236.

68. Dib-Hajj, S.D., et al. 2005. Gain-of-function mutation in $\mathrm{Na}_{v} 1.7$ in familial erythromelalgia induces bursting of sensory neurons. Brain. doi:10.1093/ brain/awh514

69. Fertleman, C.R., Rees, M., Parker, K.A., Barlow, E. and Gardiner, R.M. 2004. Identification of the gene underlying an inherited disorder of pain sensation. Program no. 197 presented at the 54th Annual Meeting of the American Society of Human Genetics.
October 26-30. Bethesda, Maryland, USA. http:// www.ashg.org/genetics/ashg04s/index.shtml.

70. Nassar, M.A., et al. 2004. Nociceptor-specific gene deletion reveals a major role for Nav1.7 (PN1) in acute and inflammatory pain. Proc. Natl. Acad. Sci. U. S. A. 101:12706-12711.

71. Weiss, L.A., et al. 2003. Sodium channels SCN1A, SCN2A and SCN3A in familial autism. Mol. Psychiatry. 8:186-194.

72. Kim, J., et al. 2004. Calmodulin mediates Ca2+ sensitivity of sodium channels. J. Biol. Chem. 279:45004-45012.

73. Splawski, I., et al. 2004. Ca(V)1.2 calcium channel dysfunction causes a multisystem disorder including arrhythmia and autism. Cell. 119:19-31.

74. Wasserman, D., Geijer, T., Rozanov, V., and Wasserman, J. 2005. Suicide attempt and basic mechanisms in neural conduction: relationships to the SCN8A and VAMP4 genes. Am. J. Med. Genet. B Neuropsychiatr. Genet. 133:116-119.

75. Do, M.T., and Bean, B.P. 2004. Sodium currents in subthalamic nucleus neurons from Nav1.6-null mice. J. Neurophysiol. 92:726-733.

76. Maurice, N., Tkatch, T., Meisler, M., Sprunger, L.K., and Surmeier, D.J. 2001. D1/D5 dopamine receptor activation differentially modulates rapidly inactivating and persistent sodium currents in prefrontal cortex pyramidal neurons. J. Neurosci. 21:2268-2277.

77. Raman, I.M., Sprunger, L.K., Meisler, M.H., and Bean, B.P. 1997. Altered subthreshold sodium currents and disrupted firing patterns in Purkinje neurons of Scn8a mutant mice. Neuron. 19:881-891.

78. Yan, H., Yuan, W., Velculescu, V.E., Vogelstein, B. and Kinzler, K.W. 2002. Allelic variation in human gene expression. Science. 297:1143.

79. Claes, L., et al. 2003. De novo SCN1A mutations are a major cause of severe myoclonic epilepsy of infancy. Hum. Mutat. 21:615-621.

80. Guerrini, R., and Aicardi, J. 2003. Epileptic encephalopathies with myoclonic seizures in infants and children (severe myoclonic epilepsy and myoclonicastatic epilepsy). J. Clin. Neurophysiol. 20:449-461.

81. Nabbout, R., et al. 2003. Spectrum of SCN1A mutations in severe myoclonic epilepsy of infancy. Neurology. 60:1961-1967.

82. Oguni, H., et al. 2005. Severe myoclonic epilepsy in infancy: clinical analysis and relation to SCN1A mutations in a Japanese cohort. Adv. Neurol. 95:103-117.

83. Ohmori, I., et al. 2003. Is phenotype difference in severe myoclonic epilepsy in infancy related to SCN1A mutations? Brain Dev. 25:488-493.

84. Sugawara, T., et al. 2002. Frequent mutations of SCN1A in severe myoclonic epilepsy in infancy. Neurology. 58:1122-1124.

85. Spampanato, J., Aradi, I., Soltesz, I., and Goldin, A.L. 2004. Increased neuronal firing in computer simulations of sodium channel mutations that cause generalized epilepsy with febrile seizures plus. J. Neurophysiol. 91:2040-2050.

86. Kohrman, D.C., Smith, M.R., Goldin, A.L., Harris, J., and Meisler, M.H. 1996. A missense mutation in the sodium channel Scn8a is responsible for cerebellar ataxia in the mouse mutant jolting. J. Neurosci. 16:5993-5999.

87. Kearney, J.A., et al. 2002. Molecular and pathological effects of a modifier gene on deficiency of the sodium channel Scn8a (Na(v)1.6). Hum. Mol. Genet. 11:2765-2775.

88. Akopian, A.N., et al. 1999. The tetrodotoxin-resistant sodium channel SNS has a specialized function in pain pathways. Nat. Neurosci. 2:541-548. 